\title{
Nonlinear Tracking Control of Underactuated Surface Vessel
}

\author{
Wenjie Dong and Yi Guo
}

\begin{abstract}
We consider in this paper the tracking control problem of an underactuated surface vessel. Based on the cascaded structure of tracking error dynamics, we present two new tracking controllers using Barbalat's lemma and backstepping techniques under mild assumptions on the reference trajectory. The closed-loop systems achieve global asymptotical and exponential tracking respectively. Simulation results show that the proposed control laws are effective.
\end{abstract}

\section{INTRODUCTION}

Control of underactuated surface vessels has attracted much attention recently. The state of the underactuated surface vessel cannot be stabilized to the origin by any smooth pure state feedback control law due to Brockett's necessary condition ([3]). Furthermore, the model of the underactuated surface vessel is not drift-less, and the control methods developed for stabilizing nonholonomic systems cannot be directly used to solve the stabilization problem of this system. With the effort of researchers, several stabilizing control laws have been developed. Interested readers may refer to [20][7][17][19][15][5][1].

The tracking control problem of underactuated surface vessel has also been studied. In [2] and [9], output tracking control is discussed based on feedback linearization and Lyapunov theory. In [1] and [18], global practical tracking controllers are presented, where the tracking errors are made to converge to a neighborhood of the origin. In [14], a $\mathcal{K}$-exponential tracking controller is proposed based on the cascaded structures of the tracking error dynamics. In [10], an asymptotical tracking controller and an exponential tracking controller are proposed. The asymptotical tracking controller is derived with the passivity-based $L_{g} V$-type control strategy and the exponential tracking controller is proposed by combining the cascade structure of the tracking error dynamics and backstepping technique.

In this paper, we consider the tracking control problem of an underactuated surface vessel. Under mild assumptions, we propose new tracking controllers with the aid of the cascade structure of the closed-loop system. In order to propose the controllers, we first apply a nonlinear coordinate transformation to the system, and the tracking error dynamics is derived with a cascaded structure. Then an asymptotical tracking controller is designed using Barbalat's lemma and backstepping. Furthermore, in order to improve the convergence rate of the tracking error, an exponential tracking controller is proposed by fully exploiting the

Department of Electrical and Computer Engineering, University of Central Florida cascaded structure of the tracking errors, which ensures that the tracking error globally exponentially converges to zero. Compared with existing controllers, our controllers make the tracking errors converge to zero asymptotically or exponentially. Good tracking performance is guaranteed under mild assumptions made on the reference trajectories.

The rest of the paper is organized as follows. In section 2, the problem under study is stated. In Section 3, an asymptotical tracking controller is designed. Then an exponential tracking control law is presented in Section 4. In Section 5 , simulation results are shown. Finally, we conclude the paper in Section 6.

\section{PROBlem STATEMENT}

Consider the tracking controller problem of an underactuated surface vessel. The vessel has two propellers which are the force in surge and the control torque in yaw. Following the results in [8], the kinematics of the system can be written as

$$
\left[\begin{array}{c}
\dot{x} \\
\dot{y} \\
\dot{\psi}
\end{array}\right]=\left[\begin{array}{ccc}
\cos \psi & -\sin \psi & 0 \\
\sin \psi & \cos \psi & 0 \\
0 & 0 & 1
\end{array}\right]\left[\begin{array}{l}
u \\
v \\
r
\end{array}\right]
$$

where $(x, y)$ denotes the coordinate of the center of mass of the surface vessel in the earth-fixed frame, $\psi$ is the orientation of the vessel, and $u, v$ and $r$ are the velocities in surge, sway and yaw, respectively. Assume that

1) the environment forces due to wind, currents and waves can be neglected in the model,

2) the inertia, added mass and damping matrices are diagonal.

The dynamics of the surface vessel is described as ([8])

$$
\left\{\begin{array}{l}
\dot{u}=\frac{m_{22}}{m_{11}} v r-\frac{d_{11}}{m_{11}} u+\frac{1}{m_{11}} \tau_{1}, \\
\dot{v}=-\frac{m_{11}}{m_{22}} u r-\frac{d_{22}}{m_{22}} v \\
\dot{r}=\frac{m_{11}-m_{22}}{m_{33}} u v-\frac{d_{33}}{m_{33}} r+\frac{1}{m_{33}} \tau_{2},
\end{array}\right.
$$

where $m_{i i}>0$ are given by the vessel inertia and the added mass effects, $d_{i i}>0$ are given by the hydrodynamic damping, $m_{i i}$ and $d_{i i}$ are assumed to be constant. $\tau_{1}$ and $\tau_{2}$ are the surge control force and the yaw control moment, respectively.

Given a bounded feasible reference trajectory $\left(x_{d}, y_{d}, \theta_{d}, u_{d}, v_{d}, r_{d}\right)$ with reference input $\left(\tau_{1 d}, \tau_{2 d}\right)$ 
which satisfies

$$
\left\{\begin{array}{l}
\dot{x}_{d}=u_{d} \cos \psi_{d}-v_{d} \sin \psi_{d} \\
\dot{y}_{d}=u_{d} \sin \psi_{d}+v_{d} \cos \psi_{d} \\
\dot{\psi}_{d}=r_{d} \\
\dot{u}_{d}=\frac{m_{22}}{m_{11}} v_{d} r_{d}-\frac{d_{11}}{m_{11}} u_{d}+\frac{1}{m_{11}} \tau_{1 d} \\
\dot{v}_{d}=-\frac{m_{11}}{m_{22}} u_{d} r_{d}-\frac{d_{22}}{m_{22}} v_{d} \\
\dot{r}_{d}=\frac{m_{11}-m_{22}}{m_{33}} u_{d} v_{d}-\frac{d_{33}}{m_{33}} r_{d}+\frac{1}{m_{33}} \tau_{2 d}
\end{array}\right.
$$

the tracking control problem under study is to design a control law for system (1)-(2) such that

$$
\begin{aligned}
\lim _{t \rightarrow \infty}\left(x-x_{d}\right) & =0, \lim _{t \rightarrow \infty}\left(y-y_{d}\right)=0, \\
\lim _{t \rightarrow \infty}\left(\psi-\psi_{d}\right) & =0, \lim _{t \rightarrow \infty}\left(u-u_{d}\right)=0, \\
\lim _{t \rightarrow \infty}\left(v-v_{d}\right) & =0, \lim _{t \rightarrow \infty}\left(r-r_{d}\right)=0 .
\end{aligned}
$$

For the tracking problem, there are several controllers proposed in [9], [2], [14], [10], [18], [1]. In the following sections, we propose two new controllers: an asymptotical tracking controller and an exponential tracking controller. Due to space limit, proofs are omitted.

\section{Asymptotical Tracking Controller}

To facilitate the controller design, we transform (1)-(2) into a suitable form. Applying the following state and input transformations ([15]):

$$
\begin{aligned}
& \left\{\begin{array}{l}
z_{1}=x \cos \psi+y \sin \psi \\
z_{2}=v \\
z_{3}=-x \sin \psi+y \cos \psi+\frac{m_{22}}{d_{22}} v, \\
z_{4}=\psi \\
z_{5}=r \\
z_{6}=-\frac{m_{11}}{d_{22}} u-z_{1},
\end{array}\right. \\
& \left\{\begin{array}{l}
w_{1}=\frac{m_{11}-m_{22}}{m_{33}} u v-\frac{d_{33}}{m_{33}} r+\frac{\tau_{2}}{m_{33}} \\
w_{2}=\left(\frac{d_{11}}{d_{22}}-1\right) u-z_{3} z_{5}-\frac{\tau_{1}}{d_{22}},
\end{array}\right.
\end{aligned}
$$

we have

$$
\left\{\begin{array}{l}
\dot{z}_{1}=-\frac{d_{22}}{m_{11}} z_{1}-\frac{d_{22}}{m_{11}} z_{6}+z_{3} z_{5}-\frac{m_{22}}{d_{22}} z_{2} z_{5} \\
\dot{z}_{2}=-\frac{d_{22}}{m_{22}} z_{2}+\frac{d_{22}}{m_{22}} z_{5}\left(z_{1}+z_{6}\right) \\
\dot{z}_{3}=z_{6} z_{5} \\
\dot{z}_{4}=z_{5} \\
\dot{z}_{5}=w_{1} \\
\dot{z}_{6}=w_{2} .
\end{array}\right.
$$

Similarly, by the transformation

$$
\left\{\begin{aligned}
z_{1 d} & =x_{d} \cos \psi_{d}+y_{d} \sin \psi_{d}, \\
z_{2 d} & =v_{d}, \\
z_{3 d} & =-x_{d} \sin \psi_{d}+y_{d} \cos \psi_{d}+\frac{m_{22}}{d_{22}} v_{d}, \\
z_{4 d} & =\psi_{d}, \\
z_{5 d} & =r_{d}, \\
z_{6 d} & =-\frac{m_{11}}{d_{22}} u_{d}-z_{1 d}, \\
w_{1 d} & =\frac{m_{11}-m_{22}}{m_{33}} u_{d} v_{d}-\frac{d_{33}}{m_{33}} r_{d}+\frac{\tau_{2 d}}{m_{33}}, \\
w_{2 d} & =\left(\frac{d_{11}}{d_{22}}-1\right) u_{d}-z_{3 d} z_{5 d}-\frac{\tau_{1 d}}{d_{22}},
\end{aligned}\right.
$$

we have

$$
\left\{\begin{aligned}
\dot{z}_{1 d} & =-\frac{d_{22}}{m_{11}} z_{1 d}-\frac{d_{22}}{m_{11}} z_{6 d}+z_{3 d} z_{5 d}-\frac{m_{22}}{d_{22}} z_{2 d} z_{5 d} \\
\dot{z}_{2 d} & =-\frac{d_{22}}{m_{22}} z_{2 d}+\frac{d_{22}}{m_{22}} z_{5 d}\left(z_{1 d}+z_{6 d}\right) \\
\dot{z}_{3 d} & =z_{5 d} z_{6 d} \\
\dot{z}_{4 d} & =z_{5 d} \\
\dot{z}_{5 d} & =w_{1 d} \\
\dot{z}_{6 d} & =w_{2 d} .
\end{aligned}\right.
$$

Define the tracking error to be

$$
\begin{aligned}
e= & {\left[e_{1}, e_{2}, e_{3}, e_{4}, e_{5}, e_{6}\right]^{T}=\left[z_{1}-z_{1 d}, z_{2}-z_{2 d},\right.} \\
& \left.z_{3}-z_{3 d}, z_{4}-z_{4 d}, z_{5}-z_{5 d}, z_{6}-z_{6 d}\right]^{T} .
\end{aligned}
$$

We have

$$
\begin{aligned}
& \left\{\begin{aligned}
\dot{e}_{1}= & -\frac{d_{22}}{m_{11}} e_{1}-\frac{d_{22}}{m_{11}} e_{6}+e_{3} e_{5}+z_{3 d} e_{5} \\
& +z_{5 d} e_{3}-\frac{m_{22}}{d_{22}}\left(e_{2} e_{5}+z_{2 d} e_{5}+e_{2} z_{5 d}\right)_{(10)} \\
\dot{e}_{2}= & -\frac{d_{22}}{m_{22}} e_{2}+\frac{d_{22}}{m_{22}}\left(e_{1} e_{5}+z_{1 d} e_{5}\right. \\
& \left.+e_{1} z_{5 d}+e_{5} e_{6}+e_{5} z_{6 d}+z_{5 d} e_{6}\right)
\end{aligned}\right. \\
& \left\{\begin{aligned}
\dot{e}_{3}= & e_{5} e_{6}+e_{5} z_{6 d}+z_{5 d} e_{6} \\
\dot{e}_{4}= & e_{5} \\
\dot{e}_{5}= & w_{1}-w_{1 d} \\
\dot{e}_{6}= & w_{2}-w_{2 d}
\end{aligned}\right.
\end{aligned}
$$

Lemma 1: Using the transformations (4), (5), and (7), $\lim _{t \rightarrow \infty} e_{i}=0(1 \leq i \leq 6)$ implies that $(x, y, \psi, u, v, r)$ asymptotically converges to $\left(x_{d}, y_{d}, \psi_{d}, u_{d}, v_{d}, r_{d}\right)$,

System (10)-(11) is a cascaded system. An important issue in controlling cascaded systems is to design a control law to avoid peaking phenomenon [21]. However, for system (10)-(11), the peaking phenomenon never appears if the sub-system (11) is asymptotically stable.

Lemma 2: For the cascaded system (10)-(11), if $\left(x_{d}, y_{d}, u_{d}, v_{d}, r_{d}\right)$ is bounded and $e_{i}(3 \leq i \leq 6)$ globally converge to zero, then $e_{1}$ and $e_{2}$ globally converge to zero.

Based on Lemma 2, we propose a global tracking controller in two steps using backstepping [11]. In the first step, we consider the stabilization problem of the subsystem $\left(e_{3}, e_{4}, e_{6}\right)$. Assuming $e_{5}$ is a virtual control, we design $e_{5}$ and $w_{2}$ such that $e_{3}, e_{4}$ and $e_{6}$ globally asymptotically 
converge to zero. The result of the first step is stated in the following lemma.

Lemma 3: In (11), if $z_{5 d}$ and $z_{6 d}$ are bounded and $\lim _{t \rightarrow \infty} z_{5 d}(t) \neq 0$, let

$$
\begin{aligned}
e_{5} & =\chi \\
w_{2} & =-k_{2}\left(e_{6}-\alpha\right)-z_{5 d} e_{3}+w_{2 d}+\dot{\alpha}
\end{aligned}
$$

where

$$
\begin{aligned}
& \alpha=-k_{3} z_{5 d} e_{3}, \\
& \chi=-k_{4} e_{4}-k_{4} e_{3}\left(e_{6}+z_{6 d}\right),
\end{aligned}
$$

constants $k_{i}>0(2 \leq i \leq 4)$, then $e_{3}, e_{4}$ and $e_{6}$ asymptotically converge to zero, respectively.

In the second step, we design an actual control $w_{1}$ so that $e_{3}, e_{4}, e_{5}$ and $e_{6}$ asymptotically converge to zero with the aid of the result in Lemma 3. We have the following result.

Lemma 4: For system (11), if $z_{5 d}$ and $z_{6 d}$ are bounded and $\lim _{t \rightarrow \infty} z_{5 d}(t) \neq 0$, the control law

$$
\begin{gathered}
w_{1}=-k_{1}\left(e_{5}-\chi\right)-e_{3}\left(e_{6}+z_{6 d}\right)-e_{4}+w_{1 d}+\dot{\chi} \\
w_{2}=-k_{2}\left(e_{6}-\alpha\right)-z_{5 d} e_{3}+w_{2 d}+\dot{\alpha}
\end{gathered}
$$

globally asymptotically stabilize $e_{3}, e_{4}, e_{5}$ and $e_{6}$ to the origin, where constants $k_{i}>0(1 \leq i \leq 4), \alpha$ and $\chi$ are defined in (14) and (15).

With the aid of the results in Lemma 2 and Lemma 4, we have the following theorem.

Theorem 1: For system (6), if $\left(x_{d}, y_{d}, \psi_{d}, u_{d}, v_{d}, r_{d}\right)$ is bounded and $\lim _{t \rightarrow \infty} r_{d}(t) \neq 0$, then the control law (16)(17) ensures that $(x, y, \psi, u, v, r)$ globally asymptotically converges to $\left(x_{d}, y_{d}, \psi_{d}, u_{d}, v_{d}, r_{d}\right)$.

In Theorem 1, a global tracking controller is proposed with the assumption that $\left(x_{d}, y_{d}, \psi_{d}, u_{d}, v_{d}, r_{d}\right)$ is bounded and $\lim _{t \rightarrow \infty} r_{d}(t) \neq 0$. In the controller, there are only four control parameters $k_{1}, k_{2}, k_{3}$ and $k_{4}$. In order to make the state of the closed-loop system asymptotically converge to the given trajectory, they are only needed to be positive. Generally, large values of $k_{i}(1 \leq i \leq 4)$ make the tracking errors converge to zero fast. However, controller (16)-(17) does not guarantee that the convergence rate of the tracking error is exponential. In order to make the tracking errors converge to zero fast, an exponential tracking controller is proposed in the next section.

\section{Exponential Tracking CONTROLler}

Based on the transformation (4)-(5), we have the following lemma.

Lemma 5: For the cascaded system (10)-(11), if $\left(x_{d}, y_{d}, \psi_{d}, u_{d}, v_{d}, r_{d}\right)$ is bounded and (11) is globally exponentially stable, then (10)-(11) is globally exponentially stable.

By Lemma 5, it only needs to consider the system (11). For (11), we have the following result.
Lemma 6: For system (11), if $\left(x_{d}, y_{d}, \psi_{d}, u_{d}, v_{d}, r_{d}\right)$ is bounded and $r_{d}$ satisfies

$$
\int_{0}^{t} r_{d}^{2}(s) d s \geq \delta t,(\forall t \geq 0)
$$

where constant $\delta>0$, the control inputs

$$
\begin{aligned}
w_{1}= & w_{1 d}-\left(k_{1}+k_{2}\right) e_{5}-k_{1} k_{2} e_{4} \\
w_{2}= & w_{2 d}-k_{3} \dot{z}_{5 d} e_{3}-k_{3} z_{5 d} \dot{e}_{3}-k_{4} e_{6} \\
& -\left(k_{3} k_{4}+1\right) z_{5 d} e_{3}-e_{3} e_{5}
\end{aligned}
$$

ensure $e_{i}(3 \leq i \leq 6)$ globally exponentially converge to zero, where control parameters $k_{i}>0(1 \leq i \leq 4)$ and $k_{1} \neq k_{2}$.

By Lemma 5 and Lemma 6, we have the following theorem.

Theorem 2: For system (6), if $\left(x_{d}, y_{d}, \psi_{d}, u_{d}, v_{d}, r_{d}\right)$ is bounded and $r_{d}$ satisfies (18), control law (19)-(20) ensure $e_{i}(1 \leq i \leq 6)$ globally exponentially converge to zero, where control parameters $k_{i}>0(1 \leq i \leq 4)$ and $k_{1} \neq k_{2}$.

In the control laws (19) and (20), the control parameters are $k_{i}(1 \leq i \leq 4)$. If $k_{i}>0(1 \leq i \leq 4)$ and $k_{1} \neq k_{2}$, the states of the closed-loop system (10) globally exponentially converge to zero. The exponential convergence rate of $e_{i}(3 \leq i \leq 6)$ can be adjusted by the control parameters $k_{i}(1 \leq i \leq 4)$. The exponential convergence rate of $e_{1}$ and $e_{2}$ depends on $d_{22}, m_{11}, m_{22}$ and the exponential convergence rate of $e_{3}, e_{4}, e_{5}$ and $e_{6}$. It should be noted that the assumption on $r_{d}$ is not strict.

In this paper, we propose two tracking controllers. The first one in Theorem 1 makes the tracking errors asymptotically converge to zero, while the second one in Theorem 2 makes the tracking errors exponentially converge to zero. However, the assumptions made on the reference trajectories in Theorem 1 are less conservative than that in Theorem 2.

Several tracking controllers are proposed in literature. In [1] and [18], global practical tracking controllers are presented. The tracking errors are made to converge to a neighborhood of the origin. While our exponential controller makes the tracking errors exponentially converge to zero. In [14], a $\mathcal{K}$-exponential tracking controller is proposed based on the cascaded structures of the tracking error dynamics. In [10], an exponential tracking controller is proposed. The exponential tracking controller is proposed by combining the cascade structure of the dynamics of the tracking errors and the backstepping technique. The assumptions made on the reference trajectories are the same as those in Theorem 2. Advantages of our proposed controller over some previous results in literature include: 1) It can make the tracking errors exponentially converge to zero; 2) The assumptions made on the reference trajectories are less conservative and easily verified; 3) The controller is simple and the control parameters are easily chosen.

\section{Simulation}

In this section, we study the effectiveness of the proposed control laws by simulation. Consider an underactuated sur- 
face vessel with the model parameters [20]: $m_{11}=200 \mathrm{~kg}$, $m_{22}=250 \mathrm{~kg}, m_{33}=80 \mathrm{~kg}, d_{11}=70 \mathrm{~kg} / \mathrm{s}, d_{22}=100 \mathrm{~kg} / \mathrm{s}$, $d_{33}=50 \mathrm{~kg} / \mathrm{s}$. Assume the initial condition of the system is $(x(0), y(0), \psi(0), u(0), v(0), r(0))=(30,-1,0.2,0,0,0)$.

The reference trajectory to be tracked is similar to that in [14]. Assume the initial reference state are $x_{d}(0)=-2 m$, $y_{d}(0)=-20 \mathrm{~m}, \psi_{d}(0)=0 \mathrm{rad}, u_{d}(0)=1 \mathrm{~m} / \mathrm{s}, v_{d}(0)=$ $-0.1 \mathrm{~m} / \mathrm{s}, r_{d}(0)=0.05 \mathrm{rad} / \mathrm{s}$ and $u_{d}(t)=1 \mathrm{~m} / \mathrm{s}, r_{d}(t)=$ $0.05 \mathrm{rad} / \mathrm{s}$, the reference trajectory $\left(x_{d}, y_{d}, \psi_{d}, u_{d}, v_{d}, r_{d}\right)$ can be generated by (3) with proper $\tau_{1 d}$ and $\tau_{2 d}$.

We first use the control law (16)-(17) to system (1)-(2). The control parameters are chosen as $k_{1}=1, k_{2}=1.5$, $k_{3}=10$ and $k_{4}=2$. Figs $1-8$ are simulation results. Figs 1-6 show the tracking errors of the closed-loop system converge to zero. Fig. 7 shows the desired trajectory and the actual trajectory of the vessel in $X-Y$ plane. Fig. 8 shows that the two control inputs are bounded. The simulation results verify the result in Theorem 1 .

In the second simulation, the exponential stabilizing law (19) and (20) is applied to system (6) with the model parameters and initial condition stated above. In the control law, we choose $k_{1}=1.2, k_{2}=1, k_{3}=10, k_{4}=1.5$. Figs 9-17 are simulation results. Figs 9-14 show that the given desired trajectories and the response of each state. They show that the states of the closed-loop system converge to the desired trajectories. Furthermore, Fig. 15 shows that the tracking errors exponentially converge to zero because logarithm of absolute value of each tracking error decrease linearly. Fig. 16 shows the desired trajectory and the actual trajectory of the vessel in $X-Y$ plane. Fig. 17 shows that the control inputs are bounded.

From the simulation, the two proposed control laws all make the tracking error converge to zero. Since the first control law does not guarantee an exponential convergence rate of the tracking error of the closed-loop system, the tracking performance may be not good as one desires. The second control law guarantees that the tracking error exponentially converge to zero. Therefore, the performance of the tracking error system is satisfactory.

\section{CONCLUSION}

In this paper, the tracking control problem of an underactuated surface vessel is considered. New asymptotical tracking controller and exponential tracking controller are proposed with the aid of the cascaded structure of the error dynamics, Barbalat's lemma, and the backstepping technique. They can make the tracking errors converge to zero asymptotically or exponentially. Simulation results show the proposed controllers are effective. The ideas developed in this paper can be applied to design controllers of other underactuated systems.

\section{REFERENCES}

[1] A. Behal, D. M. Dawson, W. E. Dixon, and Y. Fang, "Tracking and regulation control of an underactuated surface vessel with nonintegrable dynamics," IEEE Trans. on Automatic Control, vol.47, no.3, 2002, pp.495-500.
[2] S. Berge, K. Ohtsu, and T. Fossen, "Nonlinear control of ships minimizing the position tracking errors," Modeling Identification Control, vol.20, pp.141-147, 1999.

[3] R. Brockett, "Asymptotic stability and feedback stabilization," in Differential Geometric Control Theory, R. Brockett, R. Millman, and H. Sussmann, Eds. Boston, MA: Birkhauser, 1983.

[4] F. Bullo, "Stabilization of relative equilibria for underactuated systems on Riemannian manifolds," Automatica, vol.36, no.12, 2000, pp.1819-1834.

[5] F. Bullo, N. E. Leonard, and A. D. Lewis, "Controllability and motion algorithms for underactuated Lagrangian systems on Lie groups," IEEE Trans. Automatic Control, vol.45, 2000, pp.1437-1454.

[6] W.E. Dixon, D.M. Dawson, F. Zhang, and E. Zergeroglu, "Global exponential tracking control of a mobile robot system via a PE condition," IEEE Transactions on Systems, Man, and Cybernetics, vol.30, pp.129-142, 2000.

[7] I. Fantoni, R. Lozano, F. Mazenc, and K.Y. Pettersen, "Stabilization of a nonlinear underactuated hovercraft," Int. J. Robust Nonlinear Control, vol.10, no.8, 2000, pp.645-654.

[8] T.I. Fossen, Guidance and Control of Ocean Vehicles, New York: Wiley, 1994.

[9] J.-M. Godhavn, "Nonlinear tracking of underactuated surface vessels," Proc. 35th Conf. Decision Control, Kobe, Japan, December 1996, pp.975-980.

[10] Z.P. Jiang, "Global tracking control of underactuated ships by Lyapunov's direct method," Automatica, vol.38, 2002, pp.301-309.

[11] M. Krstic, I. Kanellakopoulos, and P. Kokotovic, Nonlinear and Adaptive Control Design. New York, NY: Wiley, 1995.

[12] H.K. Khalil, Nonlinear Systems. 2nd Edition, Prentice-Hall, Englewood Cliffs, NJ, 1996.

[13] I. Kolmanovsky and N.H. McClamroch,"Developments in nonholonomic control systems," IEEE Control System Magazine, vol.15, no.6, 1995, pp.20-36.

[14] E. Lefeber, K. Y. Pettersen, and H. Nijmeijer, "Tracking control of an underactuated ship," IEEE Trans. on Control systems Technology, vol.11, no.1, 2003, pp.52-61.

[15] F. Mazenc, K.Y. Pettersen, and H. Nijmeijer, "Global uniform asymptotic stabilization of an underactuated surface vessel," IEEE Trans. on Automatic Control, vol.47, no.10, 2002, pp.1759-1762.

[16] K.Y. Pettersen, "Exponential stabilization of underactuated vehicles," Ph.D. thesis, Norwegian University of Science and Technology, 1996.

[17] K.Y. Pettersen and O. Egeland, "Exponential stabilization of an underactuated surface vessel," Proc. 35th IEEE Conf. Decision and Control, Kobe, Japan, 1996, pp.967-971.

[18] K.Y. Petterson and H. Nijmeijer, "Global practical stabilization and tracking for an underactuated ship-A combined averaging and backstepping approach," Proc. IFAC Conf. System Structure Control, Nantes, France, July 1998. pp.59-64.

[19] K.Y. Pettersen and O. Egeland, "Time-varying exponential stabilization of the position and attitude of an underactuated autonomous underwater vehicle," IEEE Trans. on Automatic Control, vol.44, no.1, 1999, pp.112-115.

[20] M. Reyhanoglu, "Control and stabilization of an underactuated surface vessel," Proc. 35th IEEE Conf. Decision and Control, Kobe, Japan, 1996, pp.2371-2376.

[21] H.J. Sussmann and P.V. Kokotovic, "The peaking phenomenen and the global stabilization of nonlinear systems," IEEE Trans. on Automatic Control, vol.36, no.4, 1991, pp.424-440.

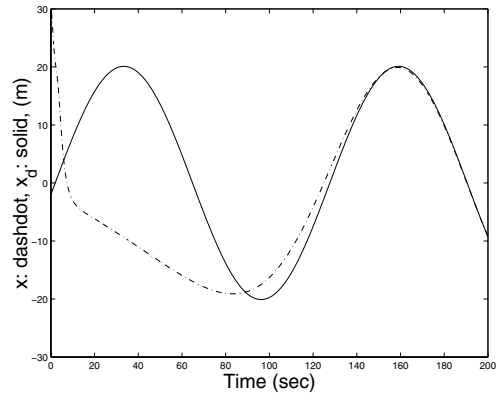

Fig. 1. Response of $x$ and $x_{d}$ 


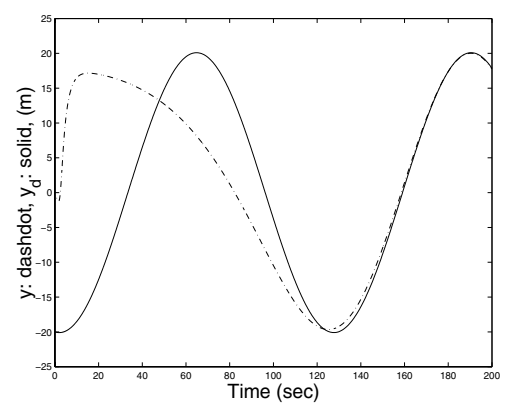

Fig. 2. Response of $y$ and $y_{d}$

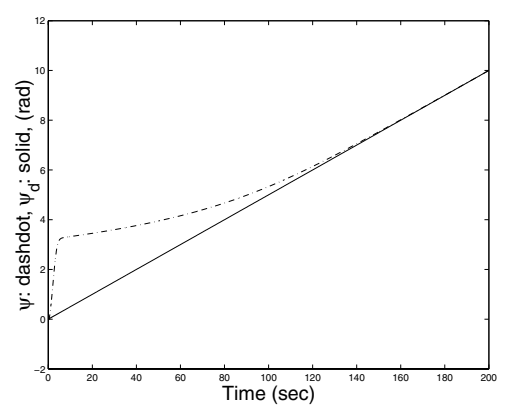

Fig. 3. Response of $\psi$ and $\psi_{d}$

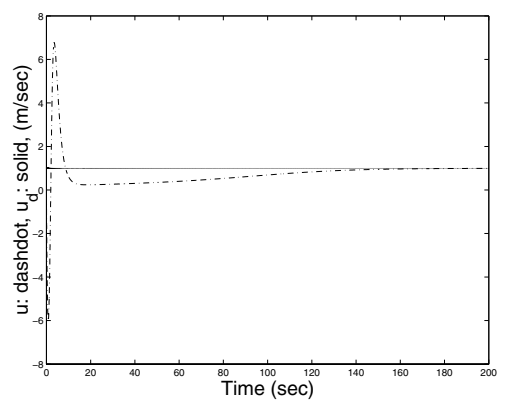

Fig. 4. Response of $u$ and $u_{d}$

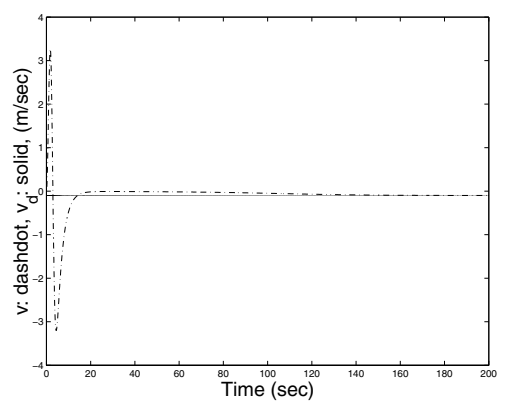

Fig. 5. Response of $v$ and $v_{d}$

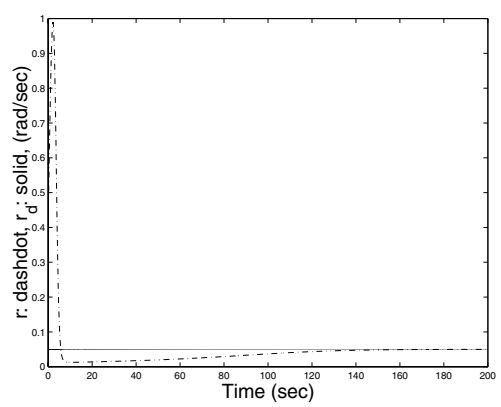

Fig. 6. Response of $r$ and $r_{d}$

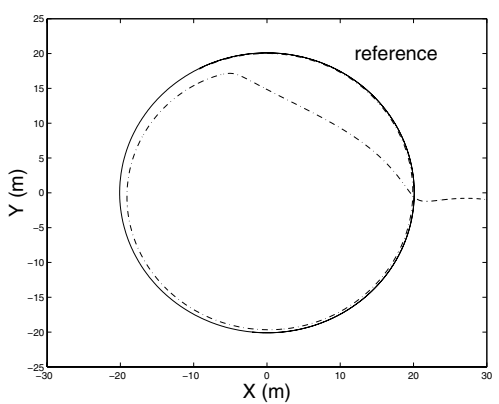

Fig. 7. Trajectory in $X-Y$ plane

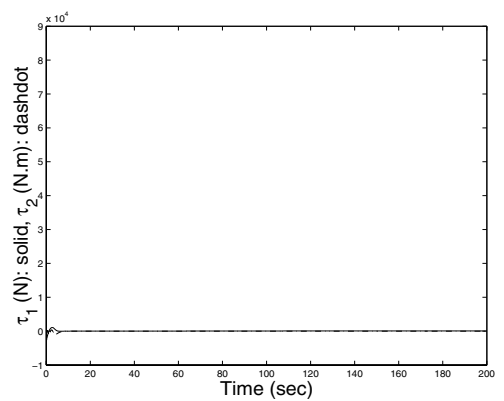

Fig. 8. Control inputs

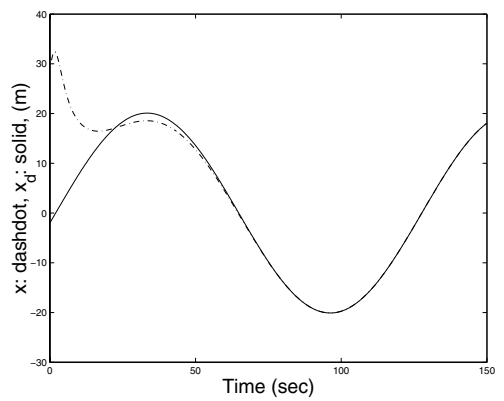

Fig. 9. Response of $x$ and $x_{d}$ 


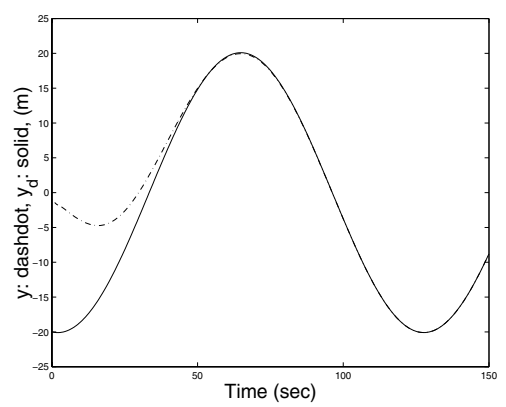

Fig. 10. Response of $y$ and $y_{d}$

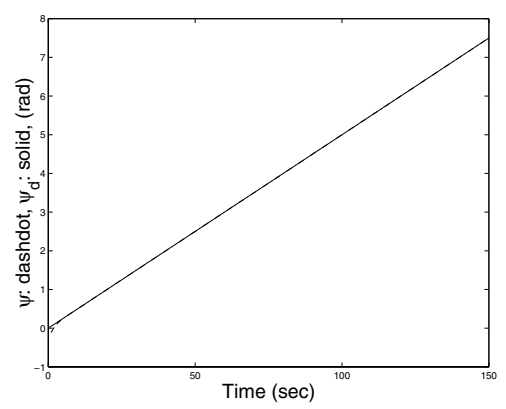

Fig. 11. Response of $\psi$ and $\psi_{d}$

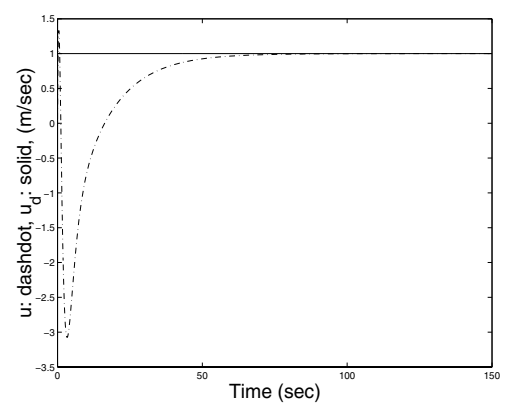

Fig. 12. Response of $u$ and $u_{d}$

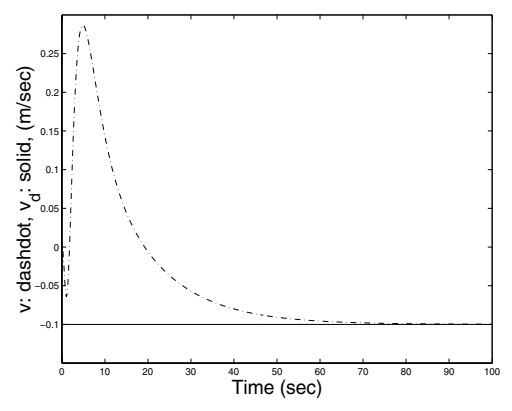

Fig. 13. Response of $v$ and $v_{d}$

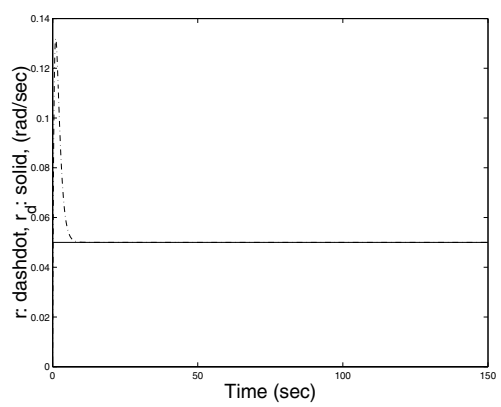

Fig. 14. Response of $r$ and $r_{d}$

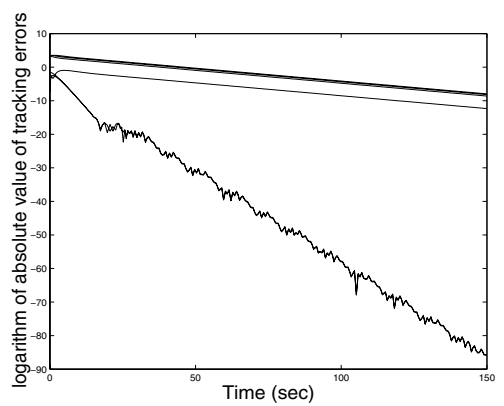

Fig. 15. Logarithm of absolute value of tracking errors

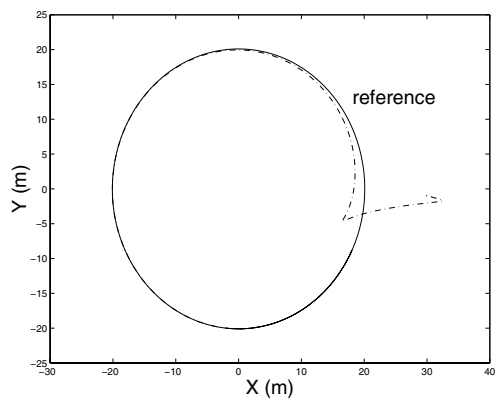

Fig. 16. Trajectory in $X-Y$ plane

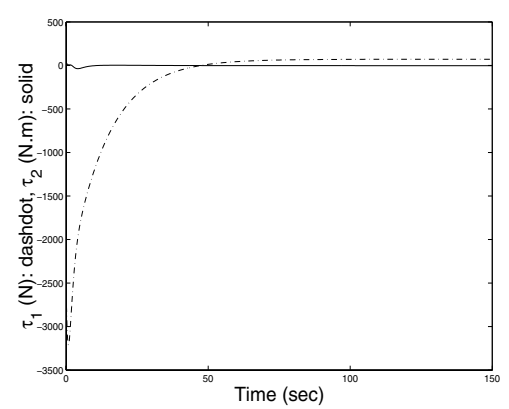

Fig. 17. Control inputs 